Pacific Journal of Mathematics

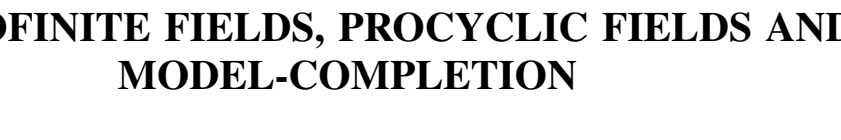




\title{
PSEUDOFINITE FIELDS, PROCYCLIC FIELDS AND MODEL-COMPLETION
}

\author{
Allan Adler and Catarina Kiefe
}

\begin{abstract}
In this paper, it is shown that the theory of pseudofinite fields is, with respect to a suitable language, the model completion of the theory of procyclic fields. Also, procyclic fields are characterized as the class of relatively algebraically closed subfields of pseudofinite fields.
\end{abstract}

The first two sections of this paper contain some basic definitions and results necessary for an understanding of the main theorems. These are stated at the end of $\S 1$. As is well-known, a model completion of a theory is not determined by the models of the theory alone, but also by the language in which the theory is formulated. This makes the choice of a model-completion rather arbitrary. The advantage of the particular language adopted here for the theory of procyclic fields is that not only does one obtain a model-completion of the theory (Theorem I) but one can recover the models of the theory as the class of all substructures of the models of the model-completion for which the defining axioms of the extended language hold (Theorem 2). This is proved using the results of Ax [1,2] and Jarden [3]. It is worth remarking that in this language, the theory of pseudofinite fields has elimination of quantifiers [Kiefe, 4]. Also, the authors wish to acknowledge that the key idea in the proof of Theorem 2 was inspired by A. Robinson [6].

1. Let $\tau$ be a similarity type, $L_{\tau}$ the first-order language of type $\tau$. Given two theories $\Sigma_{1}$ and $\Sigma_{2}$ in $L_{\tau}$, we recall that $\Sigma_{2}$ is called the model-completion of $\Sigma_{1}$ if the following three conditions hold:

(i) any model of $\Sigma_{2}$ is a model of $\Sigma_{1}$.

(ii) any model of $\Sigma_{1}$ can be embedded (as a substructure) into a model of $\Sigma_{2}$.

(iii) if $\mathfrak{H}_{1}$ and $\mathfrak{H}_{2}$ are models of $\Sigma_{2}$ having $\mathfrak{S}$ as a common substructure, and if $\mathfrak{S}$ is a model of $\Sigma_{1}$, then $\mathfrak{A}_{1}$ and $\mathfrak{A}_{2}$ are elementarily equivalent in the language of $\left(5\right.$, i.e., in $L_{\delta .5}$ we have $\left\langle\mathfrak{H}_{1},\{c\}_{c \in|\in|}\right\rangle \equiv$ $\left\langle\mathfrak{A}_{2},\{c\}_{c \in \mid \varepsilon_{1}}\right\rangle$.

It is well-known that model-completion, when it exists, is unique. However, there is no reason to expect model-completion to be "independent of the language", i.e., "preserved under extensions by definitions". In fact, this is not the case for the theories dealt with in this paper. 
2. Let $K$ be a field: $\tilde{K}$ will denote the algebraic closure of $K$, $G(\tilde{K} / K)$ the Galois group of $\tilde{K}$ over $K$. We recall the following definition:

Definition 1. A field $K$ is called procyclic if it is perfect and has at most one extension of each finite degree.

Ordinary field language will be denoted $L_{\tau}$ and consists of the first-order language with equality, two constant symbols (0 and 1) and three funtion symbols $(+, \cdot,-)$. It is easy to check that procyclic fields form an $E C_{\Delta}$-class with respect to this language; the theory of procyclic fields, i.e., the set of sentences of $L_{\tau}$ satisfied by all procyclic fields will be denoted $\Sigma_{1}$.

Definition 2. A field $K$ is called pseudofinite if it is an infinite model of the theory of finite fields, i.e., if $K$ is infinite and every sentence of $L_{\tau}$ which holds in every finite field also holds in $K$.

Although not crucial to this paper, it is interesting to bear in mind that a purely algebraic description of the class of pseudofinite fields exists: a field $F$ is pseudofinite iff $F$ is perfect, has exactly one extension of each finite degree and every nonempty absolutely irreducible variety over $F$ has an $F$-rational point (cf. [2]).

We will denote by $\Sigma_{2}$ the theory of pseudofinite fields, i.e., the set of sentences in $L_{\tau}$ which hold for every pseudofinite field. Pseudofinite fields again form an $E C_{\Delta}$-class and in [2], J. Ax gives a recursive axiomatization of $\Sigma_{2}$.

It is now trivial to check that every pseudofinite field is procyclic (since every finite field is). On the other hand, Condition (iii) of the definition of model-completion fails, since $\Sigma_{2}$ is not model-complete (e.g., [2, p. 256]). Let us then introduce for every positive integer $n$ an $n+1$-ary predicate symbol $\psi_{n} ; L_{\tau}$, now denotes the first-order language obtained by adjoining the predicate sysmbols $\left\{\psi_{n}\right\}_{n \in \omega}$ to $L_{\tau^{\prime}}$. We now consider the following extensions by definitions of $\Sigma_{1}$ and $\Sigma_{2}$ :

$$
\Sigma_{\imath}^{\prime}=\Sigma_{1} \cup\left\{\psi_{n}\left(x_{0}, \cdots, x_{n}\right) \leftrightarrow \exists y\left(x_{n} y^{n}+\cdots+x_{0}=0\right) \mid n \in \omega\right\} \quad(i=1,2) .
$$

The new axioms will be called the defining axioms for the extended language (cf. Introduction).

Thus if $F \mid=\Sigma_{i}^{\prime}(i=1,2)$ and $a_{0}, \cdots, a_{n} \in F, \psi_{n}\left(a_{0}, \cdots, a_{n}\right)$ holds in $F$ iff the polynomial $a_{0}+a_{1} y+\cdots+a_{n} y^{n} \in F[y]$ has a root in $F$.

Observe that all we have done is to require "submodel" to mean "relatively algebraically closed submodel" $[4$, p. 34, Lemma 17]. Similarly, a substructure of a model of $\Sigma_{2}^{\prime}$ is relatively algebraically closed iff it satisfies the defining axioms for the extended language.

We now claim the main result: 
THEOREM 1. $\Sigma_{2}^{\prime}$ is the model-completion of $\Sigma_{1}$.

In order to prove it, we have to check the three conditions of the model-completion definition:

(i) is trivial, as mentioned before.

(ii) in [4, p. 34, Theorem 3] it is shown that $\Sigma_{2}^{\prime}$ admits elimination of quantifiers; hence, by [7, p. 63, Theorem 13.11], $\Sigma_{2}^{\prime}$ is substructurecomplete, a condition stronger than the one we want to establish.

(ii) in the present case, we can rephrase this condition as the following:

THEOREM 2. Every procyclic field is isomorphic to a relatively algebraically closed subfield of a pseudofinite field.

So, to establish Theorem 1, all we need is to prove Theorem 2.

3. Proof of Theorem 2. Let $K$ be a procyclic field; let $p$ denote the characteristic of $K$ ( $p=0$ or $p$ prime). Let $\mathbf{F}_{p}$ denote the prime field of $K$ ( $\mathbf{F}_{0}$ just denotes the rational numbers). We break up the proof into four cases:

Case 1. $K$ is a field of absolute numbers, i.e., $K$ is algebraic over $\mathbf{F}_{p}$.

Case 2. $K$ has finite nonzero transcendence degree over $\mathbf{F}_{p}$.

Case 3. $K$ has countably infinite transcendence degree over $\mathbf{F}_{p}$.

Case 4. $K$ has uncountable transcendence degree over $\mathbf{F}_{p}$ (equivalently, $K$ is uncountable).

The hard case turns out to be Case 2. In fact:

Case 1 has been dealt with by Ax [2, p. 262, Theorem 7], when he characterized the fields of absolute numbers of pseudofinite fields as exactly the procyclic absolute numbers fields.

Case 3 can be reduced to Case 2 in the following way: Let $\left\{t_{n}\right\}_{n \in \omega}$ be a transcendence basis for $K$ over $\mathbf{F}_{p}$. For each $n$, let $K_{n}$ be the relative algebraic closure of $\mathbf{F}_{p}\left(t_{0}, t_{1}, \cdots, t_{n}\right)$ in $K$. Any relatively algebraically closed subfield of a procyclic field is procyclic, hence every $K_{n}$ is procyclic. By Case 2, for each $n$, let $F_{n}$ be pseudofinite field and

$$
\varphi_{n}: K_{n} \rightarrow F_{n}
$$

a homomorphism mapping $K_{n}$ onto a relatively algebraically closed 
subfield of $F_{n}$. Let $F=\prod_{n \in \omega} F_{n} / D$ be a nonprincipal ultraproduct of the $F_{n}$ 's and

$$
\varphi: K \rightarrow F
$$

the map of $K$ : induced by the $\varphi_{n}$ 's (an element of $K$ belongs to all but a finite number of the $K_{n}$ 's). $\quad F$ is pseudofinite and it is easy to see that $\varphi(K)$ is relatively algebraically closed in $F$.

Case 4 is reduced to the previous cases as follows: by SkolemLowenheim, let $L$ be a countable field elementarily equivalent to $K$. Then, it follows by a result of Shelah [8], that $K$ and $L$ have isomorphic ultrapowers; so, in particular, $K$ is isomorphic to a relatively algebraically closed subfield of some ultrapower $L^{I} / D$ of $L$. Now, by the previous cases, $L$ is isomorphic to a relatively algebraically closed subfield of a pseudofinite field $F$; hence $L^{I} / D$ is isomorphic to a relatively algebraically closed subfield of the pseudofinite field $F^{I} / D$.

So the proof of Theorem 2 has been reduced to the following

LEMma. Let $\mathbf{F}_{p}$ denote a prime field of characteristic $p(p \geqq 0)$. Let $K$ be a procyclic algebraic extension of the field $\mathbf{F}_{p}\left(t_{1}, \cdots, t_{n}\right)$ of rational functions $(n \geqq 1)$. Then $K$ is isomorphic to a relatively algebraically closed subfield of a pseudofinite field.

To prove this lemma, we shall use the following result due to Moshe Jarden [3, p. 27, Theorem 3.5]:

"If $E$ is Hilbertian, and $E$ its algebraic closure, then for almost all $\sigma \in G(\tilde{E} / E)$ the fixed field of $\sigma$ is pseudofinite."

Two remarks are in order:

(1) Hilbertian fields are described in Lang [5, Chapter VIII]; in particular, all the fields $\mathbf{F}_{p}\left(t_{1}, \cdots, t_{n}\right)$ considered here are Hilbertian (it is crucial that we may assume $n \geqq 1$ if $p>0$ ).

(2) $G(\tilde{E} / E)$ becomes a compact group under the Krull topology, so we can define Haar measure on it; the "almost all" of the Jarden result refers to this measure. Naturally, a subset of $G(\tilde{E} / E)$ of Measure 1 is dense in $G(\tilde{E} / E)$.

Proof of Lemma. Let $E=\mathbf{F}_{p}\left(t_{1}, \cdots, t_{n}\right), n \geqq 1, \mathbf{F}_{p}$ as above. For $\sigma \in G(\tilde{E} / E)$, let $F_{\sigma}$ denote the fixed field of $\sigma$ in $\tilde{E}=\tilde{K}$. Then, by the Jarden result and the above remarks, the set

$$
H=\left\{\sigma \in G(\tilde{E} / E) \mid F_{\sigma} \text { is pseudofinite }\right\}
$$

is dense in $G(\tilde{E} / E)$. Since $K$ is procyclic, there is an automorphism $\tau$ of $\tilde{K}$ whose fixed field is $K$ (i.e., $K=F_{\tau}$ ); naturally, $\tau \in G(\tilde{E} / E)$. Since $H$ is dense in $G(\tilde{E} / E)$, let $\left\{\sigma_{n}\right\}_{n \in \omega}$ be a sequence of automorphisms such 
that $\sigma_{n} \rightarrow \tau$ and for all $n F_{\sigma_{n}}$ is pseudofinite. Let $D$ be a nonprincipal ultrafilter on $\omega$, and let $\mathscr{F}=\Pi F_{\sigma_{n}} / D$. We have a natural embedding

$$
\nu: E \rightarrow \mathscr{F} .
$$

Let $L$ denote the relative algebraic closure of $\nu(E)$ in $\mathscr{F}$ : we will prove that $K \cong L$. For this, it suffices to prove that a polynomial $f \in E[x]$ has a root in $K$ iff $f^{\nu}$ has a root in $L$ [1, p. 172, Lemma 5]: given $f \in E[x]$, let

$$
G_{f}=\left\{\sigma \in G(\tilde{E} / E) \mid F_{\sigma} \text { contains a root of } f\right\} .
$$

Claim. Under the Krull topology, $\dot{G}_{f}$ is a clopen subset of $G(\tilde{E} / E)$.

Indeed: say $F$ is the splitting field of $f$ over $E: G(\tilde{E} / F)$ is a basic open neighborhood of the identity in $G(\tilde{E} / E)$; now, if $\sigma \in G_{f}$, $\sigma G(\tilde{E} / F) \subseteq G_{f}$, so $G_{f}$ is open. And if $\sigma \notin G_{f}, \sigma G(\tilde{E} / F) \cap G_{f}=\varnothing$, so $G_{f}$ is closed. So claim is established.

But now: $f$ has a root in $K \Rightarrow G_{f}$ is a neighborhood of $\tau \Rightarrow$ all but finitely many $\sigma_{n} \in G_{f}$ (since $\sigma_{n} \rightarrow \tau$ ) $\Rightarrow f$ has a root in all but finitely many $F_{\sigma_{n}} \Rightarrow f$ has a root in $\mathscr{F} \Rightarrow f^{\nu}$ has a root in $L^{n}$ (since such a root is a fortiori algebraic over $\nu(E)$ ).

Also, since $G_{f}$ is clopen, so is $G(\tilde{E} / E)-G_{f}$. Hence: $f$ does not have a root in $K \Rightarrow G(\tilde{E} / E)-G_{f}$ is a neighborhood of $\tau \Rightarrow$ all but finitely many $\sigma_{n} \in G(\tilde{E} / E)-G_{f} \Rightarrow f$ does not have a root in all but finitely many $F_{\sigma_{n}} \Rightarrow f$ does not have a root in $L$.

\section{REFERENCES}

1. James Ax, Solving diophantine problems modulo every prime, Ann. of Math., 85 (1967), 161-183.

2. - The elementary theory of finite fields, Ann. of Math., 88 (1968), 239-271.

3. S. Shelah, Every two elementarily equivalent models have isomorphic ultrapowers, Israel J. Math.

4. Moshe Jarden, Elementary statements over large algebraic fields, doctoral thesis, The Hebrew University of Jerusalem (1969).

5. Catarina Kiefe, On the rationality of a zeta-function of a set definable over a finite field, doctoral thesis, S.U.N.Y. Stony Brook (1973).

6. Serge Lang, Diophantine geometry, Interscience Tracts No. 11 (1962).

7. G. Sacks, Saturated model theory, Benjamin (1972).

8. A. Robinson, Nonstandard Arithmetic, Bull. Amer. Math. Soc., 73 (1967), 818-843.

Received January 1, 1975. The second author was partially supported by NSF Grant No. GP-37492X1. 



\section{PACIFIC JOURNAL OF MATHEMATICS}

\section{EDITORS}

RICHARD ARENS (Managing Editor)

University of California

Los Angeles, California 90024

\section{J. Dugundu}

Department of Mathematics University of Southern California Los Angeles, California 90007

D. Gilbarg and J. Milgram Stanford University Stanford, California 94305

\section{ASSOCIATE EDITORS}
E. F. BECKENBACH
B. H. NeumanN
F. WOLF
K. YoSHIDA

\section{SUPPORTING INSTITUTIONS}

UNIVERSITY OF BRITISH COLUMBIA CALIFORNIA INSTITUTE OF TECHNOLOGY

UNIVERSITY OF CALIFORNIA

MONTANA STATE UNIVERSITY

UNIVERSITY OF NEVADA

NEW MEXICO STATE UNIVERSITY

OREGON STATE UNIVERSITY

UNIVERSITY OF OREGON

OSAKA UNIVERSITY

\author{
UNIVERSITY OF SOUTHERN CALIFORNIA \\ STANFORD UNIVERSITY \\ UNIVERSITY OF HAWAII \\ UNIVERSITY OF TOKYO \\ UNIVERSITY OF UTAH \\ WASHINGTON STATE UNIVERSITY \\ UNIVERSITY OF WASHINGTON \\ AMERICAN MATHEMATICAL SOCIETY
}

The Supporting Institutions listed above contribute to the cost of publication of this Journal, but they are not owners or publishers and have no responsibility for its contents or policies.

Mathematical papers intended for publication in the Pacific Journal of Mathematics should be in typed form or offset-reproduced (not dittoed), double spaced with large margins. Underline Greek letters in red, German in green, and script in blue. The first paragraph or two must be capable of being used separately as a synopsis of the entire paper. Items of the bibliography should not be cited there unless absolutely necessary, in which case they must be identified by author and Journal, rather than by item number. Manuscripts, in duplicate, may be sent to any one of the four editors. Please classify according to the scheme of Math. Reviews, Index to Vol. 39. All other communications should be addressed to the managing editor, or Elaine Barth, University of California, Los Angeles, California, 90024.

100 reprints are provided free for each article, only if page charges have been substantially paid. Additional copies may be obtained at cost in multiples of 50 .

The Pacific Journal of Mathematics is issued monthly as of January 1966. Regular subscription rate: $\$ 72.00$ a year (6 Vols., 12 issues). Special rate: $\$ 36.00$ a year to individual members of supporting institutions.

Subscriptions, orders for back numbers, and changes of address should be sent to Pacific Journal of Mathematics, 103 Highland Boulevard, Berkeley, California, 94708.

PUBLISHED BY PACIFIC JOURNAL OF MATHEMATICS, A NON-PROFIT CORPORATION

Printed at Jerusalem Academic Press, POB 2390, Jerusalem, Israel.

$$
\begin{gathered}
\text { Copyright } 1976 \text { Pacific Journal of Mathematics } \\
\text { All Rights Reserved }
\end{gathered}
$$




\section{Pacific Journal of Mathematics}

Vol. 62, No. 2

February, 1976

Allan Russell Adler and Catarina Isabel Kiefe, Pseudofinite fields, procyclic

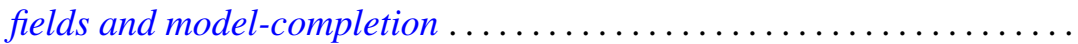

Christopher Allday, The stratification of compact connected Lie group

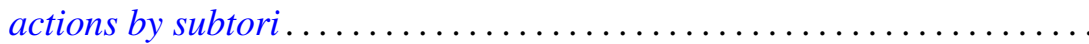

Martin Bartelt, Commutants of multipliers and translation operators .......

Herbert Stanley Bear, Jr., Ordered Gleason parts ..................

James Robert Boone, On irreducible spaces. II .....................

James Robert Boone, On the cardinality relationships between discrete

collections and open covers ............................

L. S. Dube, On finite Hankel transformation of generalized functions .......

Michael Freedman, Uniqueness theorems for taut submanifolds . . . . . . . . .

Shmuel Friedland and Raphael Loewy, Subspaces of symmetric matrices

containing matrices with a multiple first eigenvalue .............

Theodore William Gamelin, Uniform algebras spanned by Hartogs

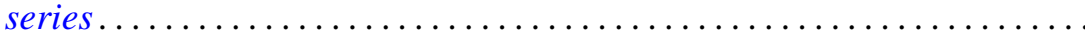

James Guyker, On partial isometries with no isometric part ............

Shigeru Hasegawa and Ryōtarō Satō, A general ratio ergodic theorem for

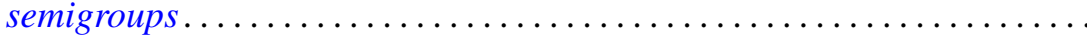

Nigel Kalton and G. V. Wood, Homomorphisms of group algebras with norm less than $\sqrt{2}$.

Thomas Laffey, On the structure of algebraic algebras...

Will Y. K. Lee, On a correctness class of the Bessel type differential operator $S_{\mu}$

Robert D. Little, Complex vector fields and divisible Chern classes ....

Kenneth Louden, Maximal quotient rings of ring extensions . .

Dieter Lutz, Scalar spectral operators, ordered $l^{\rho}$-direct sums, and the

counterexample of Kakutani-McCarthy . .

Ralph Tyrrell Rockafellar and Roger Jean-Baptiste Robert Wets, Stochastic

convex programming: singular multipliers and extended duality

singular multipliers and duality.

Edward Barry Saff and Richard Steven Varga, Geometric overconvergence of rational functions in unbounded domains ..........

Joel Linn Schiff, Isomorphisms between harmonic and P-harmonic Hardy

spaces on Riemann surfaces.

Virinda Mohan Sehgal and S. P. Singh, On a fixed point theorem of

Krasnoselskii for locally convex spaces.

Lewis Shilane, Filtered spaces admitting spectral sequence operations

Michel Smith, Generating large indecomposable continua . 